\title{
sciendo
}

\section{Recent Social Trends Among Romanian Twitter Users}

\author{
Alexandru-Răzvan FLOREA \\ Bucharest University of Economic Studies \\ alflorea@ea.com
}

\begin{abstract}
Online Social Networks have become a significant part of our quotidian life. In this paper, we aim to provide a proof of concept of how social media data can be effectively extracted, processed and analyzed with powerful open source tools like R. Moreover, we aim to build a reliable methodology for testing and validating social trends by using social media data. We used API routines to establish the connection between $R$ and Twitter, Deep Learning Models to estimate the demographics of the users, Logistic Regression Models to estimate the predispositions of the users, and Propensity Score Matching to build comparable data sets. After analyzing the Romanian Twitter users, the results of our inquiry show that most of them are relatively young and the percentage of males is significantly higher than the percentage of females. Moreover, our results confirm that facial appearances play an essential role in the popularity of an individual.
\end{abstract}

Keywords: Social Behavior, Social Media, R, Face Recognition, Data Collection, Computer Programs, Data matching

\section{Introduction}

Every person is a social nucleus able to process and spread the news. More and more individuals join online social networks. As a result, the speed of news propagation increased unprecedentedly over the past two decades. For instance, Twitter users have instant access to real-world events, and they can also spontaneously express their opinion on these events. Considering the enormous amount of social media interactions, like tweets, posts, comments, or tags, the analytical opportunity provided by these platforms is undoubtful high (MacEachren, et al., 2011) (Florea \& Roman, An ocean of migration flows data has been revealed-using social media for migration research, 2018).

Our overall aim on this paper is to prove that Social Media can be leveraged to validate local or global trends. Moreover, we want to focus on the Romanian Twitter users as there is a current need to understand better the social behavior in the emerging countries. Our methodology will combine various techniques, from descriptive statistics to advanced image processing algorithms and data set matching.

We structure our paper in 3 main sections. In the first section, we briefly present how online social networks could be leveraged in research. The second section includes the materials and techniques used in this inquiry. In the third section, we use advanced deep learning models to estimate the demographics of the Romanian Twitter users, apply complex data set matching algorithms, and provide a proof of concept for social trends validation.

\section{Literature review}

Online social activity such as posts, comments, tags, and mentions increase the spread of information. It diffuses different ideas and synchronizes the collective awareness of the 
masses and might eventually lead to online social trends (Börner, T., \& L., 2004) (Crane \& D., 2008). In other words, by substituting conventional communication portals and providing effective \& accessible environments for information transfer, online social networks have fundamentally reshaped the propagation patterns of social networks.

Twitter data unlocked significant possibilities as they can be used to answer complex questions in various fields. Researches have already found ways to make use of Twitter data. For instance, some inquiries provided ways to predict demographic characteristics using information from Twitter (Culotta, Ravi, \& Cutler, 2015). Studying demographic details can provide astonishing insights into how and why certain groups post on Twitter. In other words, a group of researchers leveraged Twitter data to understand better the marginalized crowds or even the cultural differences of certain ethnic groups (Murthy, Gross, \& Pensavalle, 2016).

Twitter data could be combined with other data sources so that astonishing results, such us happiness, education, or even obesity levels can be achieved (Mitchell, Frank, Harris, Dodds, \& Danforth, 2013). The Health Sector is of the fields which might significantly benefit from social media analysis as more and more researchers obtain resources to develop new systems to use this kind of data (Sinnenberg, et al., 2017) (Sinnenberg, DiSilvestro, \& Mancheno, 2016) (Müller \& Salathé, 2019).

However, many other fields, such as election predictions, marketing, demand planning, or human mobility, have already started using social media data. (Sanders, Gier, \& Bosch, 2016) (Luo, Cao, Mulligan, \& Lib, 2017).

There are numerous inquiries that investigated how the trends propagate among social media users. A group of researchers have found that the trends mainly rely on the crowds and influencers can only create small-scale trends (Zhang, Zhao, \& Xu, 2016).

\section{Methodology}

Our inquiry mainly relied on R, RStudio, and Microsoft Office to conduct the data collection and the statistical tests. $\mathrm{R}$ is a procedural programming language and an extraordinary instrument for generating graphics or numerical computing 1 .

In this paper, we proposed an algorithm ${ }^{2}$ structured in 10 steps (Florea \& Roman, An ocean of migration flows data has been revealed-using social media for migration research, 2018) (Florea \& Roman, The Profile of Social Media Users in Romania: INDIVIDUAL CHARACTERISTICS AND THE NUMBER OF SOCIAL CONNECTIONS, 2019) (Florea \& Roman, Using Twitter data for the research of Romanian migration flows , 2018). The first step represents the agreement with Twitter and the installation of $\mathrm{R}$ libraries. The second and third steps describe the settings preparations while the fourth, fifth, and sixth steps explain the raw data collection. The next 2 steps apply the face recognition algorithm to detect and estimate the demographics of the Romanian Twitter users. The 9th step structures and cleans the data collected in a usable format such as a data frame or a data table. The last step updates

\footnotetext{
${ }^{1} \mathrm{R}$ is a free software environment for statistical computing and graphics. It compiles and runs on a wide variety of UNIX platforms, Windows and MacOS (https://www.r-project.org/)

2 Similar Algorithm Presented in previous papers in Proceedings of IE2018, IE2019, EGE 2018 ( (Florea \& Roman, An ocean of migration flows data has been revealed-using social media for migration research, 2018) (Florea \& Roman, The Profile of Social Media Users in Romania: INDIVIDUAL CHARACTERISTICS AND THE NUMBER OF SOCIAL CONNECTIONS, 2019) (Florea \& Roman, Using Twitter data for the research of Romanian migration flows , 2018))
} 
a local data base so that the information can be processed and analyzed effectively.

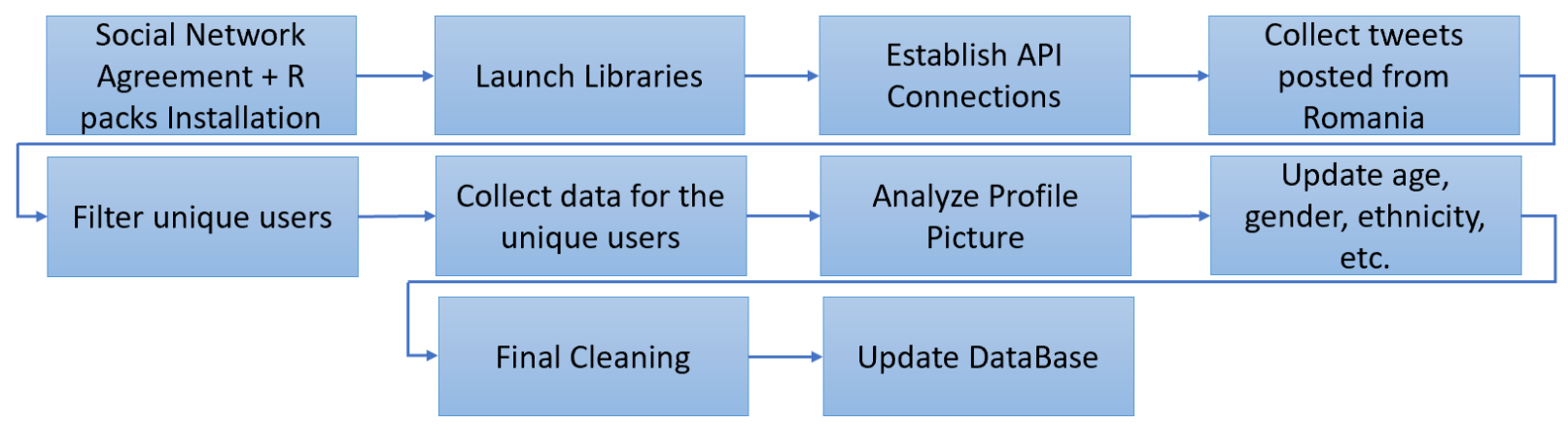

Figure 1. Algorithm Proposed for the Twitter Data Collection

Source: Graph \& Process produced by authors

The tweets used in this study were collected through the official API (Application Program Interface) ${ }^{3}$ provided by Twitter. We collected approximately 300.000 tweets posted from somewhere in Romania, from almost 60.000 unique users. This process was possible by matching the location of the tweet by latitude and longitude with the coordinates of the Romanian cities. We validated the Romanian residency of the users by interrogating their declared permanent location and identified almost 8.000 unique Romanian residents.

The next step was to estimate their age, gender, ethnicity, and appearances by using Face Recognition Technology (FRT). FRT can be widely applied in many fields. For instance, it currently contributes to international security by matching real-time images with passports photos. FRT also served as a vital component for demographic studies that used photos provided by various databases (Florea \& Roman, Using Face Recognition with Twitter Data for the Study of International, 2018). In 2015, the facial recognition technologies reached a new accuracy level of $99.65 \%$ on Labeled Faces in the Wild (LFW) data set (Schroff, Kalenichenko, \& Philbin, 2015). This inquiry leveraged the expertise and processing power of Microsoft Azure in the facial recognition sector ${ }^{4}$.

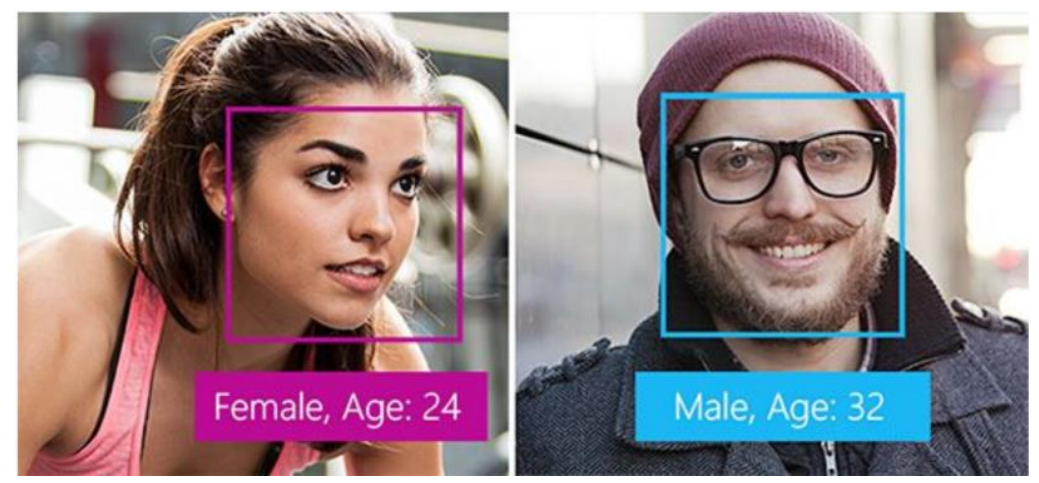

Figure 2. The accuracy of Microsoft Azure FRT

Source: Microsoft Azure Official Website

\footnotetext{
${ }^{3}$ Application programming interface

${ }^{4}$ The Azure Face API is a cognitive service that provides algorithms for detecting, recognizing, and analyzing human faces in images. (https://azure.microsoft.com/en-us/services/cognitive-services/face/)
} 
After obtaining demographic data on almost 4.000 unique Romanian Twitter users, we used Chi Squared test to initially verify if there is any association between the level of popularity and the facial appearances of men. To validate the initial hypothesis, we used the Propensity Score Matching Methodology.

We relied on the Dunbar's Number for measuring the popularity levels. A couple of decades ago, a famous British anthropologist named Robin Dunbar, discovered that the brain size of the primates is directly correlated with the size of their social groups. This stunning discovery led Dunbar to an amazing prediction: "a human being can have a maximum of 150 stable relationships." (Dunbar, 1992). The next layer mentioned by Dunbar is 500 relationships. At this level, any individual with 500 or more followers has a certain degree of popularity. For the past 20 years, a lot of investigations ${ }^{5}$ were conducted to confirm Dunbar's number and it has turned out that social networks, including the online ones, follow this theory (MacCarron, Kaski, \& Dunbar, 2016) (Gonçalves, Perra, \& Vespignani, 2011).

\section{Results and discussions}

The first purpose of our paper is to understand the characteristics of the Romanian Twitter population and the differences between them across regions.

While more than $65 \%$ of the collected users are estimated to be men, the women users tend to be slightly younger than the population of men users. The average age of Women Twitter users is 30 while the population of Men Twitter users has an average age of 35 . We also investigated the average age at the regional level and concluded that there are no significant differences among regions in terms of the age of the users. The average age of the Romanian Twitter users is between 30 and 35 in all NUTS I regions.

As expected, the most significant concentration of Twitter users is nearby the capital of the country (Bucharest-Ilfov). The North-Vest is the second region in terms of the number of collected Twitter users. On the other hand, it seems that it is less utilized in the South-East and South-Muntenia regions. However, even if the data collection includes a significant amount of Twitter users, further investigations are required to confirm the regional popularity of Twitter in Romania.

This paper aims to test a social trend that has been intensively debated in the recent years across several countries including Romania. Our research tested if having a beard or not corelates with the popularity of men. $85 \%$ of the analyzed users have less than 500 followers. Moreover, Dunbar suggests that the next layer of friendship expands up to 500 less stable relationships. Therefore, we flagged everyone who has more than 500 followers as popular Twitter user.

Running the Chi-squared test showed that having a beard is associated with high levels of popularity.

Chi-squared test:

Null hypothesis: there is no association between the two variables.

Alternative hypothesis: there is an association between the two variables.

$p$-value $<0.05=>$ the null hypothesis can be rejected for a confidence interval of $95 \%$

\footnotetext{
${ }^{5}$ MIT Technology Review: https://www.technologyreview.com/s/601369/your-brain-limits-you-to-just-five$\underline{\text { bfs/ }}$
} 


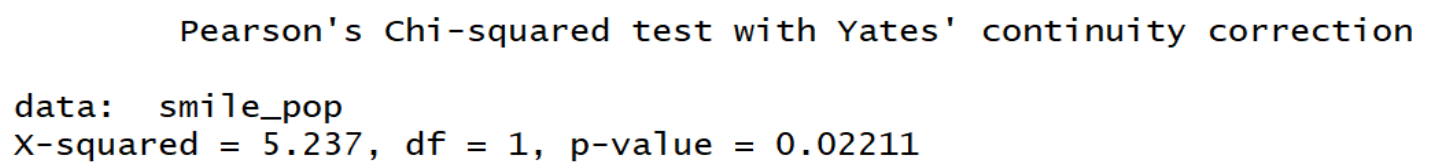

Figure 3. Popularity of Male sample; Split by facial Appearances

Source: Output produced by authors using R

However, the two populations included in the test, men with beard and men without beard, might differ in structure and therefore, the association between facial appearances and popularity might just be a spurious correlation. In other words, having a beard might not necessary be a driver for popularity. It might just be the case that the users with beard had from the beginning a predisposition to become popular.

The Propensity Score Matching (PSM) technique allowed us to remove a big portion of possible biases caused by the differences in the structure of the two populations.

First, we used a logistic regression to compute the propensity of the user's popularity. The trained logistic regression model eventually included 4 significant coefficients.

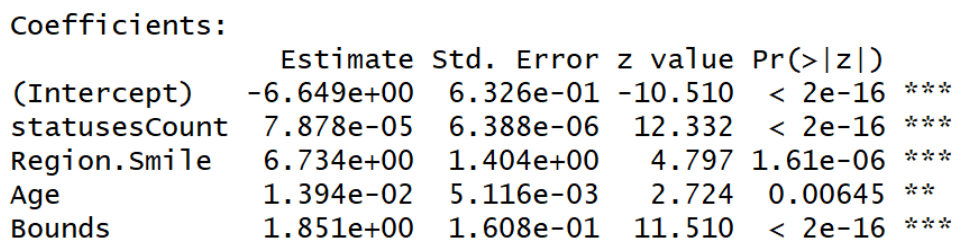

Figure 4. Coefficients of the Logistic Regression Model Source: Output produced by authors

We repeatedly trained the model and tested on different data sets and after 1000 iterations, the accuracy of the model converged to $\sim 85 \%$. In other words, our logistic model identifies the predisposition of being popular in $85 \%$ of the cases only by analyzing four variables. All four predictors are independent of the "beard/no beard" variable.

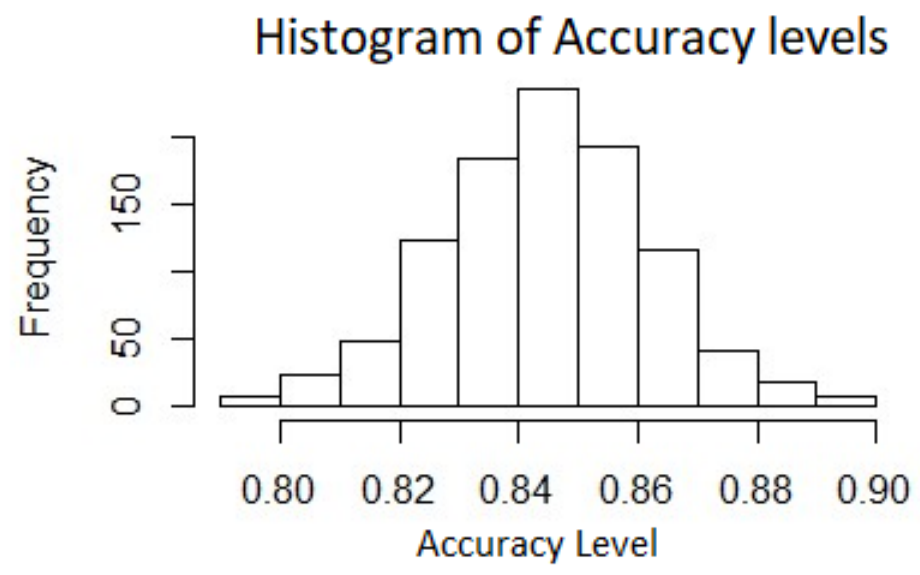

Figure 5. Accuracy of the Logistic Regression Model

Source: Output produced by authors

In the next step, we computed a sample of men without beard that followed the same propensity distribution as the sample of men with beard. After removing possible outliers 
from the two datasets, we obtained two very similar groups of twitter user in terms of the probability of being popular. The figure 6 shows that the \% popular men tend to be significantly higher in the group of men with beard.

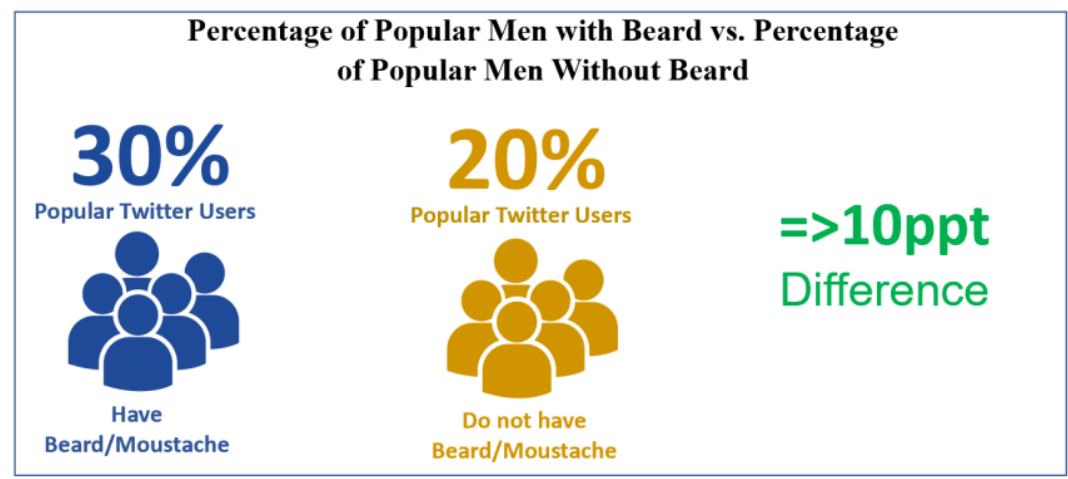

Figure 6. Percentage of Popular Men with Beard vs. Percentage of

Popular Men Without Beard

Source: Output produced by authors

The Chi Squared test applied on the matched data sets confirms that there is a significant association between beard and popularity. According to the evidences found using the PSM, this association is not driven by any known predisposition of becoming popular.

\section{Pearson's Chi-squared test with Yates' continuity correction}

data: table(data)

$\mathrm{X}$-squared $=4.0644, \mathrm{df}=1, \mathrm{p}$-value $=0.0438$

Figure X. Matched data sets: Popularity of Male sample; Split by facial Appearances Source: Output produced by authors using R

\section{Conclusion}

The enormous quantities of real-time social information have enabled scientists to investigate recent social activity as never before. The interest in utilizing datasets from online network platforms increases continuously. Even if there are numerous researches done on US social behavior, there are significantly fewer studies focused on understanding social media users in less developed countries, such as Romania. Therefore, we wanted to contribute to the collective knowledge by adding this missing piece of the big picture and analyzed the Romanian Twitter Users.

Our paper briefly explored the demographic aspects of Romanian Twitter users. We showed that online social networks could provide relevant information for the study of social trends. Moreover, our inquiry provided a robust algorithm for social data extraction and a reliable methodology for testing and validating cultural trends by analyzing social media data. The results show that having a beard play a significant role in the recent popularity of men. The main goal of the study was to offer a set of routines and methodologies that can serve as a foundation for future researches on social trends. We will continue our research on social trends by studying human mobility among Twitter users. 


\section{References}

Börner, K., T., M. J., \& L., G. R. (2004). The simultaneous evolution of author and paper networks. Proceedings of the National Academy of Sciences.

Crane, R., \& D., S. (2008). Robust dynamic classes revealed by measuring the response function of a social system. Proceedings of the National Academy of Sciences.

Culotta, A., Ravi, N. K., \& Cutler, J. (2015). Predicting the Demographics of Twitter Users from Website Traffic Data. Proceedings of the Twenty-Ninth AAAI Conference on Artificial Intelligence. Austin, Texas, USA.

Dunbar, R. I. (1992). Neocortex size as a constraint on group size in primates. Journal of Human Evolution, 22(6).

Florea, A. R., \& Roman, M. (2018). Using Twitter data for the research of Romanian migration flows. THE INTERNATIONAL ECONFERENCE "ENTERPRISES IN THE GLOBAL ECONOMY".

Florea, A. R., \& Roman, M. (2019). The Profile of Social Media Users in Romania: INDIVIDUAL CHARACTERISTICS AND THE NUMBER OF SOCIAL CONNECTIONS. Proceedinigs of the IE 2019 International Conference. Bucharest.

Florea, A., \& Roman, M. (2018). An ocean of migration flows data has been revealed-using social media for migration research. IE 2018-The 17th International Conference on Informatics in Economy, 17. Iasi, Romania.

Florea, A., \& Roman, M. (2018). Using Face Recognition with Twitter Data for the Study of International. Informatica Economică, 22(4), 31-46.

Gonçalves, B., Perra, N., \& Vespignani, A. (2011). Modeling Users' Activity on Twitter Networks: Validation of Dunbar's Number. Plos One, 6(8).

Luo, F., Cao, G., Mulligan, K., \& Lib, X. (2017). Explore spatiotemporal and demographic characteristics of human mobility via Twitter: A case study of Chicago. Applied Geography, 70, 11-25.

MacCarron, P., Kaski, K., \& Dunbar, R. (2016). Calling Dunbar's Numbers. Social Networks, 47, 151-155.

MacEachren, Robinson, Jaiswal, Pezanowski, Savelyev, Blanford, \& Mitra. (2011). Geo-Twitter Analytics: Applications in Crisis Management. Proceedings, 25th International Cartographic Conference, Paris, France.

Mitchell, L., Frank, M. R., Harris, K. D., Dodds, P. S., \& Danforth, C. M. (2013). The Geography of Happiness: Connecting Twitter Sentiment and Expression, Demographics, and Objective Characteristics of Place. Plos One , 8(5).

Müller, M. M., \& Salathé, M. (2019). Crowdbreaks: Tracking Health Trends Using Public Social Media Data and Crowdsourcing. Front Public Health.

Murthy, D., Gross, A., \& Pensavalle, A. (2016). Urban Social Media Demographics: An Exploration of Twitter Use in Major American Cities. Journal of Computer-Mediated Communication, 21(1), 33-49.

Sanders, E., Gier, M. d., \& Bosch, A. v. (2016). Using Demographics in Predicting Election Results with Twitter. International Conference on Social Informatics. Seattle, Washington, USA.

Schroff, F., Kalenichenko, D., \& Philbin, J. (2015). FaceNet: A Unified Embedding for Face Recognition and Clustering. IEEE Xplore.

Sinnenberg, L., Buttenheim, A., Padrez, K., Mancheno, C., Ungar, L., \& Merchant, R. (2017). Twitter as a Tool for Health Research: A Systematic Review. American Journal of Public Health (AJPH), 107(1).

Sinnenberg, L., DiSilvestro, C. L., \& Mancheno, C. (2016). Twitter as a Potential Data Source for Cardiovascular Disease Research. JAMA Cardiol, 1(9), 1032-1036.

Zhang, L., Zhao, J., \& Xu, K. (2016). Who creates Trends in Online Social Media: The Crowd or Opinion Leaders? Journal of Computer-Mediated Communication, 21(1), 1-16. 